\title{
ON THE ACYCLIC POINT-CONNECTIVITY OF THE n-CUBE
}

\author{
JOHN BANKS \\ Department of Mathematics \\ University of California, Santa Cruz \\ Santa Cruz, California 95064 U.S.A. \\ and \\ JOHN MITCHEM \\ Department of Mathematics and Computer Science \\ San Jose State University \\ San Jose, California 95192 U.S.A. \\ (Received October 5, 1981 and in revised form May 21, 1982)
}

ABSTRACT. The acyclic point-connectivity of a graph $G$, denoted $\alpha(G)$, is the minimum number of points whose removal from G results in an acyclic graph. In a 1975 paper, Harary stated erroneously that $\alpha\left(Q_{n}\right)=2^{n-1}-1$ where $Q_{n}$ denotes the $n$-cube. We prove that for $n>4,7 \cdot 2^{n-4} \leq \alpha\left(Q_{n}\right) \leq 2^{n-1}-2^{n-y-2}$, where $y=\left[\log _{2}(n-1)\right]$. We show that the upper bound is obtained for $n \leq 8$ and conjecture that it is obtained for all n.

KEY WORDS AND PHRASES. n-cube, acyclic point-connectivity.

1980 MATHEMATICS SUBJECT CLASSIFICATION CODES. 05C05, 05C40.

1. INTRODUCTION.

Definitions and examples for this paper can be found in Harary [1], which has a readable survey of the general area to which our results and conjectures apply. For current work, consult especially the Journal of Graph Theory and the Journal of Combinatorics, Series B. More specifically, in [2] Harary introduced the acyclic pointconnectivity of a graph $G$, denoted $\alpha(G)$, as the minimum number of points whose removal from $G$ results in an acyclic graph. He then stated without proof that $\alpha\left(K_{p}\right)=p-2$, $\alpha\left(K_{m, n}\right)=\min \{m, n\}-1$ and $\alpha\left(Q_{n}\right)=2^{n-1}-1$, where $Q_{n}$ denotes the $n-c u b e$. The first two equalities are indeed trivial; the last one is incorrect. In this paper we obtain upper bounds for $\alpha\left(Q_{n}\right)$, show that the upper bound is obtained for $n \leq 8$, and conjecture 
that the upper bound is obtained for all $\mathrm{n}$.

Before beginning the proof we introduce the following notation and definitions. For any real number $x,[x]$ denotes the greatest integer not greater than $x$. The distance between two points $v$ and $w$ is denoted by $d(v, w)$. The $n$-cube $Q_{n}$ is the graph with point set consisting of all n-tuples of $0^{\prime} s$ and $1^{\prime} s$, and two points are adjacent iff they differ in exactly one coordinate. We observe that $Q_{n}$ is bipartite and all points of $Q_{n}$ with fixed ith coordinate, $1 \leq i<n$, induce the subgraph $Q_{n-1}$. In fact, the subgraph of $Q_{n}$ induced by all points with $n$th coordinate $O(1)$ is denoted $Q_{n-1}^{\prime}\left(Q_{n-1}^{\prime \prime}\right)$

2. MAIN RESULTS.

THEOREM 1. For $n>4,7 \cdot 2^{n-4} \leq \alpha\left(Q_{n}\right) \leq 2^{n-1}-2^{n-y-2}$, where $y=\left[\log _{2}(n-1)\right]$. We begin the proof of Theorem 1 with a number of lemmas. Some of the easy proofs are omitted.

LEMPA 1. Let $v, w \in V\left(Q_{n}\right) ; d(v, w)=j$ iff $v$ and $w$ differ in exactly $j$ coordinates.

LEMMA 2. For $u, v \in V\left(Q_{n}, n>1\right.$, if $d(u, v)=2$, then $u$ and $v$ are contained in exactly one 4-cycle.

LEMMA 3. If $\alpha\left(Q_{n}\right)=t$, then $\alpha\left(Q_{n+1}\right) \geq 2 t$.

PROOF. This follows immediately from the observation that $Q_{n+1}$ contains two disjoint copies of $Q_{n}$.

LEMMA 4. $\alpha\left(Q_{2}\right)=1$ and $\alpha\left(Q_{3}\right)=3$.

PROOF. The first equality is clear and with Lemma 3 implies that $\alpha\left(Q_{3}\right) \geq 2$. Let $u, v \in V\left(Q_{3}\right)$. If $d(u, v) \leq 2(=3)$, then $Q_{3}-\{v, u\}$ contains a 4-cycle (6-cycle). Thus $\alpha\left(Q_{3}\right) \geq 3$. Equality follows from the fact that the removal of $(1,1,0),(0,1,1)$, $(1,0,1)$ from $Q_{3}$ results in a disconnected graph consisting of $K_{1}$ and $K_{1,3}$, which we denote by $K_{1} \cup K_{1,3^{\circ}}$ We also note that the five point path $P_{5}$ results from the removal of $(0,0,0),(1,1,0)$, and $(0,0,1)$ from $Q_{3}$.

LEMMA 5. The only induced acyclic subgraphs of $Q_{3}$ with order 5 are $P_{5}$ and $\mathrm{K}_{1} \cup \mathrm{K}_{1,3^{\circ}}$

PROOF. Exactly one of $\mathrm{Q}_{2}{ }^{\prime}$ and $\mathrm{Q}_{2}{ }^{\prime \prime}$ has three points of such a subgraph $\mathrm{H}$. These three points induce $P_{3}$, and since $H$ contains no cycles and is induced, it follows that $\mathrm{H}=\mathrm{P}_{5}$ or $\mathrm{K}_{1} \cup \mathrm{K}_{1,3^{\circ}}$ 
LEMMA 6. $\alpha\left(Q_{4}\right)=6$ and the resulting induced acyclic subgraph $H$ of $Q_{4}$ consists of two disjoint copies of $\mathrm{K}_{1,4^{\circ}}$

PROOF. From Lemmas 4 and 3 it follows that $\alpha\left(Q_{4}\right) \geq 6$. However, Figure 1 shows that six points may be removed to form $K_{1,4} \cup K_{1,4^{*}}$

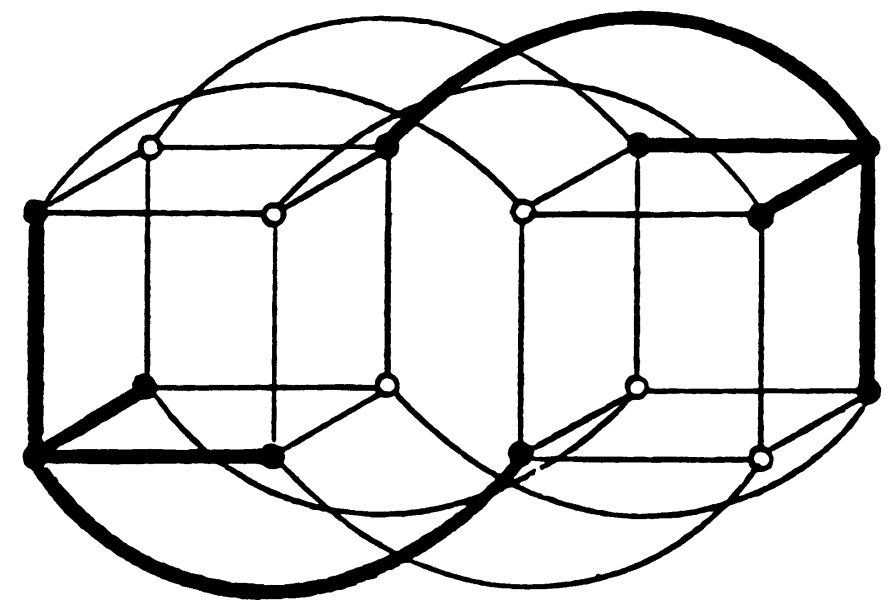

\section{Figure 1 G}

Thus $\alpha\left(Q_{4}\right)=6$, and according to Lemma 5 when $H$ is formed each of $Q_{3}{ }^{\prime}$ and $Q_{3}^{\prime \prime}$ must have three points removed forming respectively $H^{\prime}$ and $H^{\prime \prime}$. Also, $H^{\prime}$ and $H^{\prime \prime}$ are $P_{5}$ or $K_{1} \cup K_{1,3^{\circ}}$ Assume $H^{\prime}=P_{5^{\circ}}$. Now $H^{\prime}$ is adjacent with two points of $H^{\prime \prime}$ and these points must be in different components of $H^{\prime \prime}$. Thus $H^{\prime \prime}=K_{1} \cup K_{1,3}$, and points of $H^{\prime}$ are adjacent to the three points removed from $Q_{3}$ ' and the isolated point of $H^{\prime \prime}$. However, this implies that $\mathrm{H}^{\prime}$ contains $\mathrm{K}_{1,3^{\circ}}$ Thus $\mathrm{H}^{\prime}=\mathrm{H}^{\prime \prime}=\mathrm{K}_{1,3} \cup \mathrm{K}_{1}$.

Let $v_{i}\left(w_{i}\right), 1 \leq i \leq 8$, be the points of $Q_{3}{ }^{\prime}\left(Q_{3}{ }^{\prime \prime}\right)$ and let $v_{i}$ be adjacent to $w_{1}$, $1 \leq i \leq 8$. Also let $\left\{\mathrm{v}_{1}, \mathrm{v}_{2}, \mathrm{v}_{3}, \mathrm{v}_{4}\right\}$ induce $\mathrm{K}_{1,3}$ and $\mathrm{v}_{8}$ be the isolated point in $\mathrm{H}^{\prime}$. Now it can be checked that if $w_{i}, 1 \leq 1 \leq 7$, is the required point of degree three in $\mathrm{H}^{\prime \prime}$, then $\mathrm{H}$ contains a cycle. Thus ${ }_{8}$ must be the degree three point and the Lemma is proved.

LEMMA 7. $\alpha\left(Q_{5}\right)=14$. 
PROOF. According to Lemmas 6 and $3, \alpha\left(Q_{5}\right) \geq 12$. Figure 2 shows that $\alpha\left(Q_{5}\right) \leq 14$.

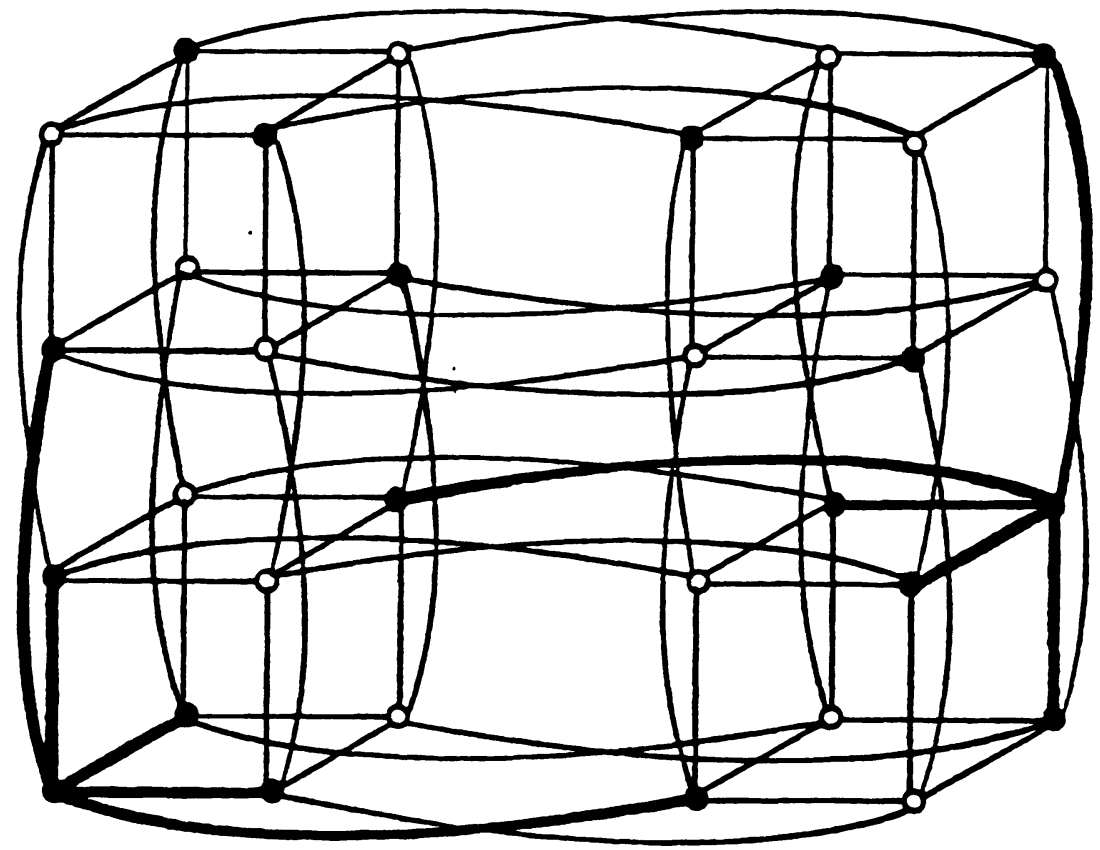

FIGURE 2 G

Assume that $\left(Q_{5}\right)=12$ or 13 , and let $H$ be an acyclic subgraph of $Q_{5}$ formed by the removal of at most 13 points. Now $Q_{5}$ may be viewed as 4 disjoint copies, say $M_{1}$, $M_{2}$, $M_{3}$, and $M_{4}$ of $Q_{3}$. It follows from Lemma 4 that exactly three points have been removed from $M_{1}, M_{2}$, and $M_{3}$, and 3 or 4 points have been removed from $M_{4}$. Furthermore, Lemma 6 implies that the darkly shaded lines of Figure 3 are contained in $\mathrm{H}$. 


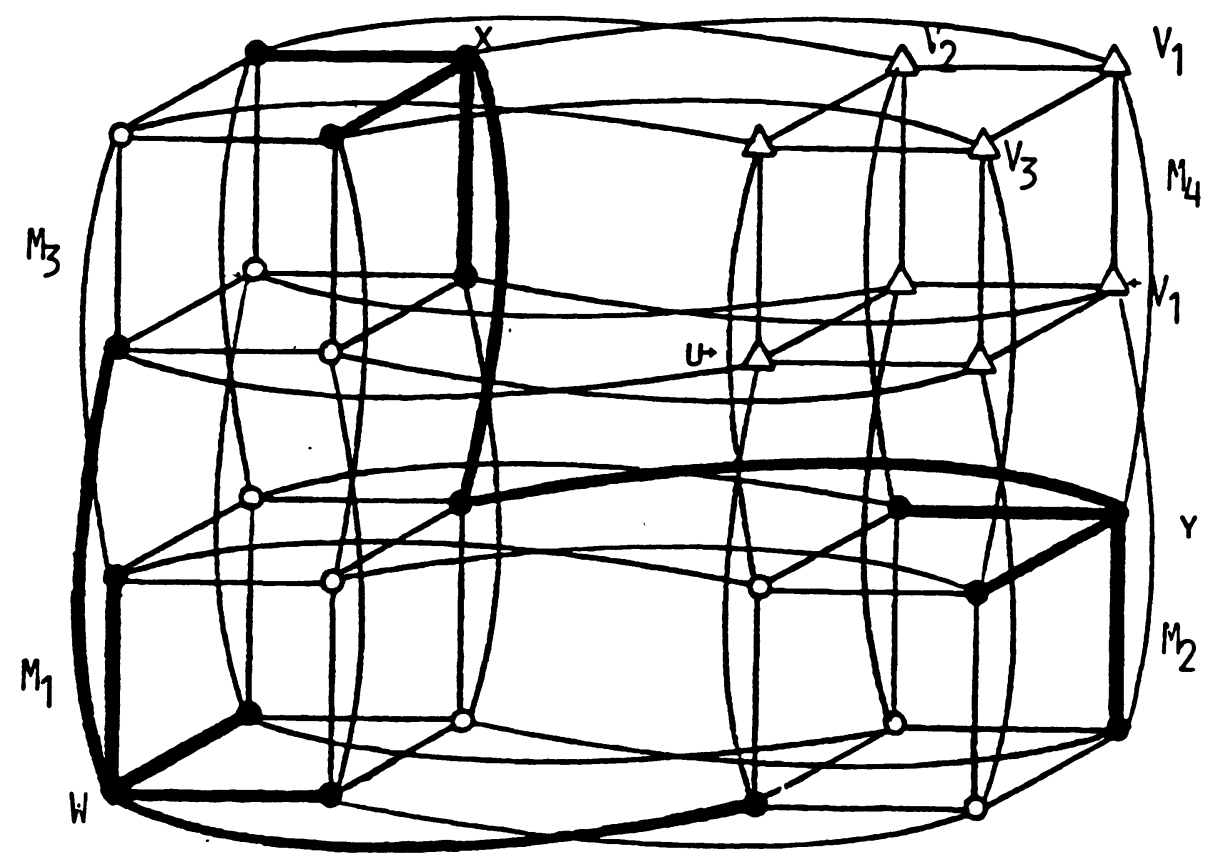

FIGLRE 3

However, as shown in Figure 3, points $v_{2}, v_{3}$, and $v_{4}$ lie in 6-cycles with $x$ and $y$, point $v_{1}$ is in a 4-cycle containing $x$ and $y$, and point $u$ is in a 4-cycle containing $w$ Hence $\alpha\left(Q_{5}\right)>14$.

Before we prove the main result two additional definitions are required. Let $1<\mathrm{k}+1<\mathrm{n}_{0}<\mathrm{n}$. Then $\theta_{1, k+1, \mathrm{n}_{0}, \mathrm{n}}$ denotes the mapping which assigns to each $\mathrm{v}$ in $V\left(Q_{n}\right)$ the point which differs from $v$ in exactly the coordinates $1, k+1, n_{0}$, and $n$. It is clear that $\theta_{1, k+1, n_{0}, n}$ is an automorphism of $Q_{n}$. Also if $\alpha_{i} \epsilon\{0,1\}$, then $\bar{\alpha}_{i}$ denotes the element in $\{0,1\}-\left\{\alpha_{i}\right\}$.

THEOREM 2. Let $\mathrm{n}>2$ and $\mathrm{y}=\left[\log _{2}(\mathrm{n}-1)\right]$. Then there exists a set of $2^{n-1}-2^{n-y-2}$ points of $Q_{n}$ whose removal results in an acyclic graph $G_{n}$ which consists of exactly $2^{\mathrm{n}-\mathrm{y}-2}$ mutually disjoint copies $\mathrm{K}_{1, \mathrm{n}}$ and $2^{\mathrm{n}-1}-\mathrm{n} 2^{\mathrm{n}-\mathrm{y}-2}$ isolated points. The set of points of degree $n$ is denoted by $C\left(G_{n}\right)$, and these points are called cluster points. $C\left(G_{n}\right)$ is a subset of one bipartite set of $Q_{n}$ and all points from the other 
bipartite set of $Q_{n}$ are contained in $G_{n}$. Furthermore, let $n_{0}=2^{y}+1$ and $n=n_{0}+k$. Then $0 \leq \mathrm{k} \leq 2^{\mathrm{y}}-1$.

If $k \neq 2^{y}-1$, let $v=\left(\alpha_{1}, \ldots, \alpha_{n}\right) \in C\left(G_{n}\right)$. Then the elements of $c_{n, v}=\left\{v_{i} \varepsilon v\left(G_{n}\right): \quad v_{i}=\left(\alpha_{1}, \alpha_{2}, \ldots, \alpha_{i-1}, a_{i}, \alpha_{i+1}, \ldots, \alpha_{n_{0}-1}, \alpha_{n_{0}}, \alpha_{n_{0}+1}, \ldots, \alpha_{n}\right)\right.$, $k+2 \leq i \leq n_{0}-1$ are isolated points of $G_{n}$. Also let $D_{n, v}=C_{n, v} u\{v\} \cup\left\{x \in V\left(G_{n}\right)\right.$ : $x$ is adjacent to $v\}$. If $u \neq v$ where $u, v \in C\left(G_{n}\right)$, then $D_{n, v} \cap D_{n, u}=\emptyset$.

PROOF. Lemmas 4,6 , and 7 verify the theorem for $n=2,3,4,5$. In order to complete the proof we show that the theorem is true for $n$, assuming it is true for $n-1$. We consider two cases.

CASE I. $\mathrm{n}=2^{\mathrm{y}}+1$

By inductive ass umption the theorem is true for $Q_{n-1}, n-1=2^{y}$. Thus there exist $2^{n-2}-2^{n-1-(y-1)-2}$ points whose removal from $Q_{n-1}$ results in graph $G_{n-1}$ which consists of $2^{n-1-(y-1)-2}=2^{n-y-2}$ mutually disjoint copies of $k_{1, n-1}$ and $2^{n-2}-(n-1)\left(2^{n-y-2}\right)=0$ isolated points. Also $C\left(G_{n-1}\right)$ is contained in a bipartite set of $Q_{n-1}$.

Let $G_{n-1}^{\prime}$ be the subgraph of $Q_{n}$ induced by all points of the form $\left(\alpha_{1}, \ldots, \alpha_{n-1}, 0\right)$ where $\left(\alpha_{1}, \ldots, \alpha_{n-1}\right) \in V\left(G_{n-1}\right)$. Let A be the bipartite set of $Q_{n}$ which contains $C\left(G_{n-1}^{\prime}\right)$ and let $B$ be the other bipartite set.

Now define $G_{n}$ as the subgraph of $Q_{n}$ induced by $B \cup C\left(G_{n-1}^{\prime}\right)$. Note $G_{n}$ has been formed by removing $2^{n-1}-2^{n-y-2}$ points from $Q_{n}$. Now $C\left(G_{n-1}^{\prime}\right)=C\left(G_{n}\right)$. Furthermore, if $u \neq v$ where $u, v \in C\left(G_{n}\right)$, the points adjacent to $v$ are disjoint from the points adjacent to $u$. Th's the $2^{n-y-2}$ copies of $k_{1, n}$ are mutually disjoint. Also $G_{n}$ contains all points of $B$ and thus $2^{n-1}-n\left(2^{n-y-2}\right)$ are isolated points.

In this case $k=0$ and $n=n_{0^{*}}$ Let $v_{i} \in C_{n, v^{*}}$ From Lemma 1 , we have that $d\left(s_{1}, v\right)=3$. Thus $v_{i} \in B$ and $v_{i} \in V\left(G_{n}\right)$. Since $v_{i}$ differs from $v$ in the nth coordinate, $v_{i} \in V\left(Q_{n-1}^{\prime \prime}\right)$. Let $w_{i}$ be the point of $Q_{n-1}^{\prime}$ adjacent to $v_{1}$. Now $d\left(w_{1}, v\right)=2$, and by Lemma $2, v$ and $w_{i}$ are in a 4-cycle of $Q_{n-1}^{\prime}$. However, all points of $B \cap V\left(Q_{n-1}^{\prime}\right)$ are in acyclic graph $G_{n}$. Thus $w_{i} \notin V\left(G_{n}\right)$ and $v_{i}$ is an isolated point.

Let $u \neq v$ be elements of $c\left(G_{n}\right)=c\left(G_{n-1}^{\prime}\right)$. Then $d(u, v) \geq 4$. Thus $c_{n, u} \cap c_{n, v}=\emptyset$, for otherwise, $d(u, y) \leq 2$. It now follows that $D_{n, u} \cap D_{n, v}=\emptyset$.

CASE II. $\mathrm{n}=2^{\mathrm{y}}+1+\mathrm{k}, 1 \leq \mathrm{k} \leq 2^{\mathrm{y}}-1$. 
Again we apply the inductive assumption on $n-1$ and obtain graph $G_{n-1}$ with the prescribed properties. Let $G_{n-1}^{\prime}$ be the subgraph of $Q_{n}$ induced by the points of the form $\left(x_{1}, \ldots, x_{n-1}, 0\right)$ where $\left(x_{1}, \ldots, x_{n-1}\right) \in V\left(G_{n-1}\right)$. Since $\theta_{1, k+1, n_{0}, n}$ is an automorphism of $Q_{n}$, it follows that $\theta_{1, k+1, n_{0}, n}\left(G_{n-1}^{\prime}\right)$, which we now denote by $G_{n-1}^{\prime \prime}$, is isomorphic to $G_{n-1}$. Thus if $u=\left(\beta_{1}, \ldots, \beta_{n-1}, 0\right) \in c\left(G_{n-1}^{\prime}\right)$, then $\bar{u}=\theta_{1, k+1, n}, n(u) \epsilon$ $C\left(G_{n-1}^{\prime \prime}\right)$. We now define $G_{n}$ as the subgraph of $Q_{n}$ induced by $V\left(G_{n-1}^{\prime}\right) \cup v\left(G_{n-1}^{\prime \prime}\right)$. It follows that $G_{n}$ was formed from $Q_{n}$ by the removal of $2\left(2^{n-2}-2^{n-y-3}\right)=2^{n-1}-2^{n-y-2}$ points. From the fact that $d(u, \bar{u})=4$ and the inductive assumption, if follows that $C\left(G_{n}\right)=C\left(G_{n-1}^{\prime}\right) \cup C\left(G_{n-1}^{\prime \prime}\right)$ is a subset of one bipartite set of $Q_{n}$ and the other bipartite set of $Q_{n}$ is contained in $V\left(G_{n}\right)$.

Let $u, v \in C\left(G_{n}\right)$ where $u \neq v$. In order to show that $D_{n, u} \cap D_{n, v}=\emptyset$ we first show that $C_{n, u} \cap C_{n, v}=\emptyset$. The latter is clear if $u \in C\left(G_{n-1}^{\prime}\right)$ and $v \in C\left(G_{n-1}^{\prime \prime}\right)$. So we suppose that $u, v \in C\left(G_{n-1}^{\prime}\right), C_{n, v}$ consists of all but one point, namely $v_{k+1}$, of $C_{n-1, v^{*}}$ Similarly $C_{n, u}$ consists of all but one point of $C_{n-1, u^{*}}$ Thus it follows from the induction assumption that $\mathrm{C}_{\mathrm{n}, \mathrm{u}} \cap \mathrm{C}_{\mathrm{n}, \mathrm{v}}=\emptyset$.

Let $v \in C\left(G_{n}\right)$ and $v_{i} \in C_{n, v^{*}}$ Then $k+2 \leq i \leq n_{0}-1$ and $v \in C\left(G_{n-1}^{\prime}\right)$ or $C\left(G_{n-1}^{\prime \prime}\right)$, say the former. By induction, $v_{i}$ was an isolated point of $G_{n-1}^{\prime}=Q_{n-1}^{\prime} \cap G_{n}$. Let $v=\theta_{1, k+1, n_{0}, n}(v)$, and $x$ be the point of $Q_{n-1}^{\prime \prime}$ which is adjacent to $v_{1}$ in $Q_{n}$. Now $\nabla \in C\left(G_{n-1}^{\prime \prime}\right)$ and $d(x, \bar{v})=2$. Thus $x \notin V\left(G_{n}\right)$, for otherwise, $G_{n-1}^{\prime \prime}$ would contain a 4cycle. Hence, $v_{i}$ is an isolated point of $G_{n}$. It now follows easily that $D_{n, u} \cap D_{n, v}=\emptyset$ when $\mathbf{u} \neq \mathbf{v}$.

Thus $G_{n}$ consists of $2\left(2^{n-y-3}\right)=2^{n-y-2}$ disjoint copies of $k_{1, n}$ and $2\left(2^{n-2}-(n-1) 2^{n-y-3}\right)-2^{n-y-2}=2^{n-1}-n 2^{n-y-2}$ isolated points. This completes the proof of Theorem 2 .

Now the upper bound of Theorem 1 follows immediately from Theorem 2 . and Theorem 1 's lower bound from Lemmas 4 and 7 . We close by stating two conjectures. Lemmas 4, 6,7 , and Theorem 1 show the first conjecture is true for $2 \leq n \leq 8$. Lemmas 4 and 6 show the second conjecture is true for $m=1$ and 2 . Our long proof for $m=3$ is omitted. It seems possible that Conjecture 2 would be useful in proving Conjecture 1. CONJECTURE 1. For $n>1, \alpha\left(Q_{n}\right)=2^{n-1}-2^{n-y-2}$ where $y=\left[\log _{2}(n-1)\right]$. 
CONJECTURE 2. If $\mathrm{n}=2^{\mathrm{m}}, \mathrm{m} \geq 1$, any maximum acyclic induced subgraph of $Q_{\mathrm{n}}$ consists of exact $1 \mathrm{y} 2^{\mathrm{n}-\mathrm{m}-1}$ disjoint copies of $\mathrm{K}_{1, \mathrm{n}}$.

\section{REFERENCES}

1. HARARY, FRANK. Graph Theory, New York, Addison-Wesley, 1969.

2. HARARY, FRANK. On Minimal Feedback Vertex Sets of a Digraph, IEEE Transactions on Circuits and Systems, $1975,839-840$. 


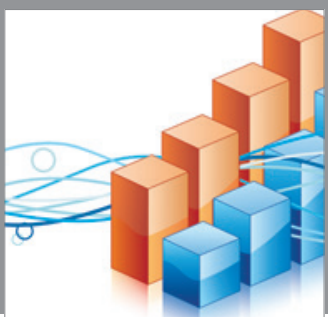

Advances in

Operations Research

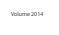

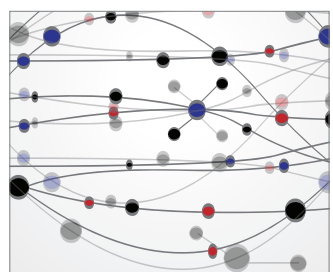

\section{The Scientific} World Journal
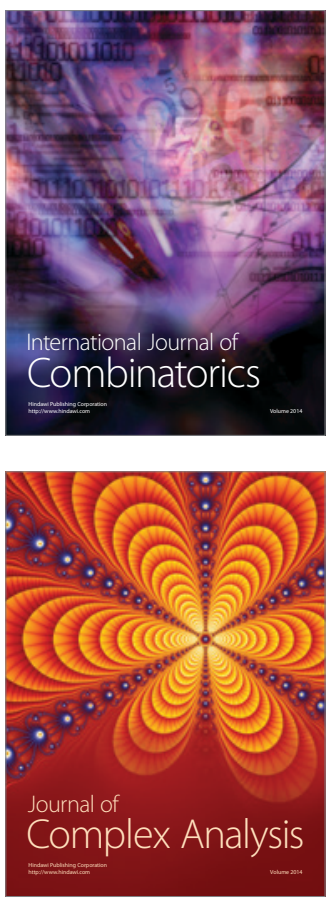

International Journal of

Mathematics and

Mathematical

Sciences
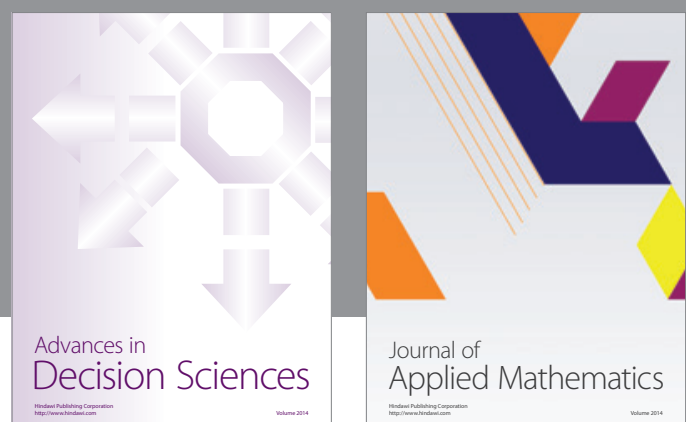

Journal of

Applied Mathematics
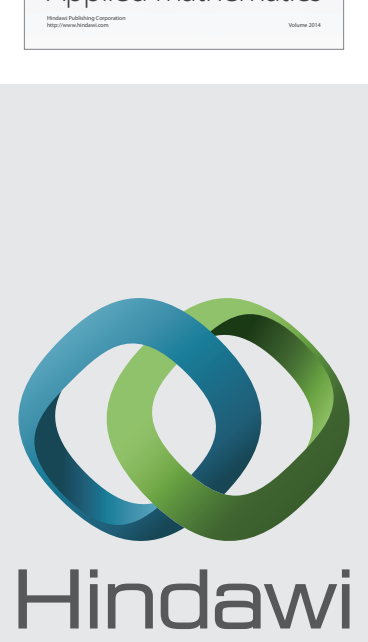

Submit your manuscripts at http://www.hindawi.com
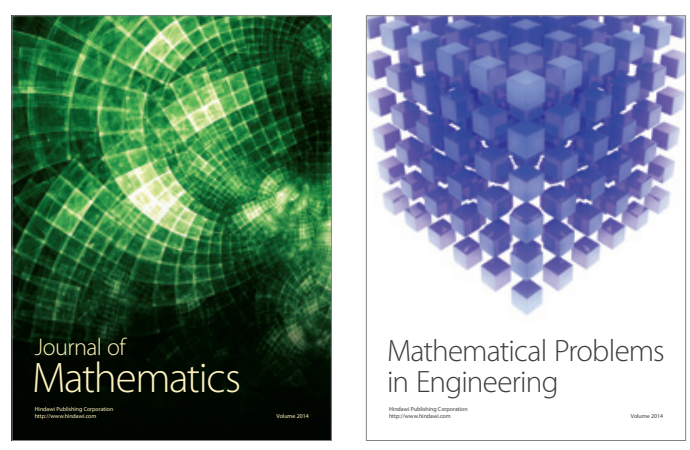

Mathematical Problems in Engineering
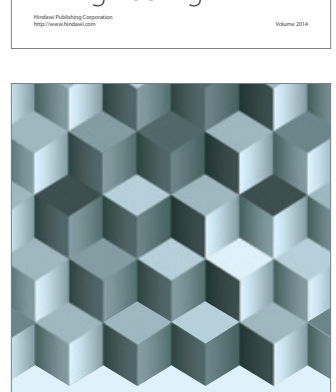

Journal of

Function Spaces
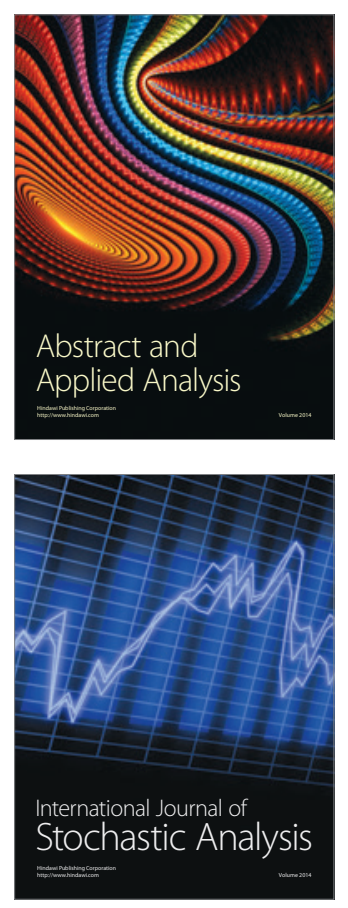

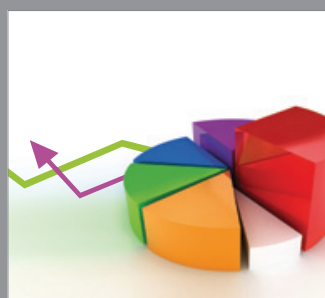

ournal of

Probability and Statistics

Promensencen
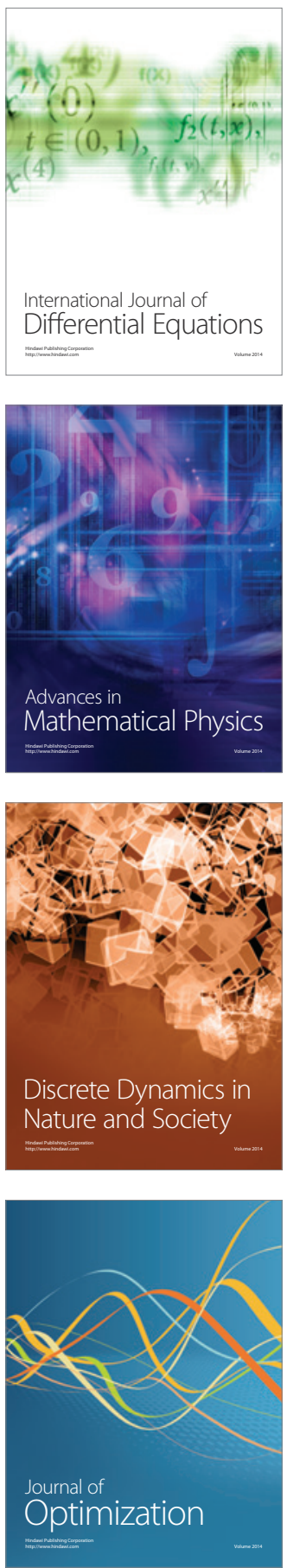